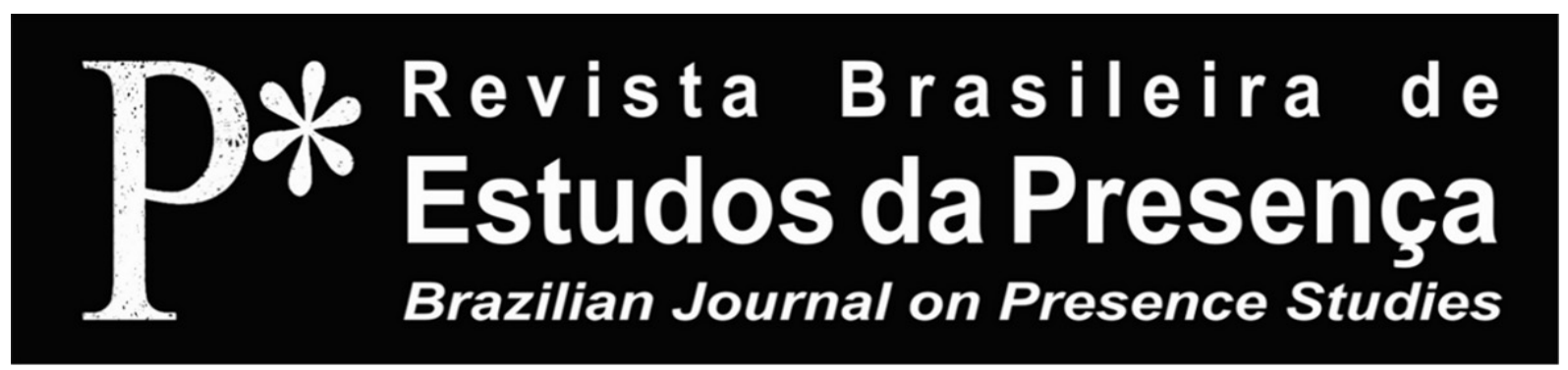

E-ISSN 2237-2660

\title{
Avoir 20 Ans en 2015: une expérience de médiation théâtrale exemplaire
}

\author{
Chantale Lepage \\ Université du Québec à Montréal - Montréal, Canada
}

RÉSUMÉ - Avoir 20 Ans en 2015: une expérience de médiation théâtrale exemplaire - Cet article présente une première analyse des retombées éducatives issues d'une expérience de médiation théâtrale qui s'est échelonnée sur cinq ans au Québec. Les données recueillies auprès des dix jeunes montréalais qui participent au projet Avoir 20 Ans en 2015 font voir la richesse et la complexité du dispositif qui a été conçu et mis en œuvre pour leur donner un accès privilégié à l'art. Des entretiens, un questionnaire et l'analyse de diverses traces témoignent de ce parcours singulier alors qu'ils se préparent à entrer de plain-pied dans la vie adulte. Les premiers résultats, même s'ils sont préliminaires, soulignent l'importance de ce type d'expérience pour des jeunes en quête d'une identité et de son affirmation.

Mots-clés: Art. Médiation Théâtrale. Culture. Adolescence. Construction Identitaire.

ABSTRACT - Being 20 Years Old in 2015: an exemplary experiment of theatrical mediation - This article presents an initial analysis of the educational impacts of a theatrical mediation experiment that was held during five years in the province of Quebec. The data collected from ten young Montrealers who participate in the project Being 20 years Old in 2015 reveals all the richness and complexity of the system that was put in place to offer them a privileged access to art. Interviews, a survey, and the analysis of different elements testify their unique journey while they are getting ready to step into adulthood. Even though the first results are only preliminary, they highlight the importance of that type of experiment for young adolescent in a quest of their identity and their affirmation. Keywords: Art. Theatrical Mediation. Culture. Adolescence. Identity Construction.

RESUMO - Ter 20 Anos em 2015: uma experiência exemplar de mediaçáo teatral Este artigo apresenta uma primeira análise dos impactos pedagógicos resultantes de uma experiência de mediaçáo teatral realizada ao longo de cinco anos no Quebec. Os dados produzidos junto a dez jovens de Montreal, participantes do projeto Ter 20 Anos em 2015, dão uma visão da riqueza e da complexidade do dispositivo criado para permitir-lhes um acesso privilegiado à arte. Entrevistas, um questionário e a análise de diversos elementos formam um testemunho dessa trajetória única justamente no momento em que esses jovens se preparam para entrar na vida adulta. Os primeiros resultados, mesmo preliminares, apontam para a importância desse tipo de experiência para jovens em busca de identidade e afirmação.

Palavras-chave: Arte. Mediação Teatral. Cultura. Adolescência. Construção Identitária. 
Plusieurs expériences esthétiques constituent des jalons importants dans le développement de certains individus. Ces expériences donnent parfois lieu à des découvertes qui favorisent la traversée de l'adolescence souvent considérée comme une zone de turbulences. L'accès à l'expérience du sensible amène les jeunes à s'ouvrir à d'autres réalités, à prendre conscience de leur héritage culturel, à se l'approprier pour mieux s'y inscrire et éventuellement y laisser, à leur tour, les marqueurs de leur génération. Ces itinéraires offrent un espace de liberté nécessaire à la construction d'une identité affirmée.

Dans son acception la plus large, [la médiation culturelle] désigne les actions d'accompagnement qui se déploient dans les espaces de production d'objets culturels et de langage produisant du sens et des liens. Elle s'incarne dans la construction de dispositifs d'interprétation des œuvres pour les publics, même néophytes, et de participation active à la vie socioculturelle pour les populations même marginalisées (Lafortune, 2012, p. 211).

À l'origine d'Avoir 20 Ans en 2015, il y a un artiste et un théâtre qui se sont associés pour proposer à des jeunes des rencontres singulières, mais aussi avec d'autres formes d'art. Ils sont invités à devenir des témoins privilégiés de certaines pratiques artistiques, et l'expérience dépasse la formation du spectateur et l'élargissement des publics. Au-delà de cet accès, les jeunes sont saisis par l'aventure humaine qui s'en dégage, mais aussi par les questionnements qu'elle suscite.

Avoir 20 Ans en 2015 est une expérience de médiation culturelle qui, même si elle se tient en dehors des murs de l'école, loge au confluent du théâtre et de l'éducation. Nous avons rencontré les jeunes participants, les avons interrogés quant à leurs parcours, leur engagement et leurs motivations, afin de prendre la mesure d'une expérience qui amorce sa cinquième et dernière année.

\section{Prendre sa Place dans le Monde}

Avoir 20 Ans en 2015 illustre de manière probante comment certains jeunes peuvent avoir accès à des expériences artistiques. Cet accès passe par la fréquentation des œuvres puisque les jeunes qui y participent ont été le public de plusieurs spectacles et évènements. Ils ont pu approfondir certaines questions en discutant de ces œuvres avec d'autres pour en construire collectivement le sens et, par 
conséquent, mieux comprendre le rôle et la fonction de l'art. Ils ont également été à même d'observer l'influence de certains artistes ou d'œuvres dans l'évolution des sociétés qui les ont vus naître. Cet accès aux œuvres passe en outre par des rencontres signifiantes avec des créateurs ou des artisans. Dans le cadre du projet, ces derniers ont joué un rôle déterminant dans la transmission de leurs pratiques artistiques et des processus qu'ils expérimentent.

Depuis 2011, cinquante jeunes issus de la francophonie font partie du projet Avoir 20 Ans en 2015. Ces jeunes proviennent de cinq villes: Montréal (Canada), Nantes (France), Mons et Namur (Belgique) et La Réunion (DROM). Dix Montréalais, cinq filles et cinq garçons, ont été sélectionnés parmi la centaine de jeunes qui avait postulé à la suite d'une invitation lancée aux élèves des écoles secondaires. Pour poser leur candidature, ils devaient se présenter à travers une ouvre: un texte, une chanson, une vidéo ou un collage, etc. Cette réalisation a permis de faire une première sélection de candidats qui ont ensuite passé un entretien. Ils devaient parler de leurs préoccupations, de leurs rêves, de leurs motivations à s'engager dans ce type de projet qui allait se poursuivre pendant cinq ans.

En concevant le projet, Wajdi Mouawad, auteur et metteur en scène, voulait d'une part «[...] replacer Sophocle au cœur du politique ${ }^{1}$ » et "[...] faire rêver ces jeunes citoyens pour qu'ils puissent mesurer l'immensité du monde et ouvrir le champ des possibles» (Bélair, 2011, p. B7). Animé par la perspective de retransmettre ce qu'il avait reçu, il a imaginé un projet tel qu'il aurait souhaité qu'on lui en propose un à quinze ans, alors qu'il était lui-même désorienté. Il voulait donner à ces jeunes la possibilité de rêver en les conviant à une grande aventure: «Voyager avec des amis dans des lieux extraordinaires avec du théâtre, avec de l'art» (Bilodeau, 2013a, 2013b, n.p.).

\section{Franchir le Cap de l'Adolescence}

S’ouvrir au théâtre, à la culture, pour franchir le cap de l'adolescence et entrer dans la vie adulte, est un rituel de passage pour le moins singulier à proposer à des jeunes de quinze ans qui, sans équivoque, ont répondu à l'appel. Leurs motivations sont multiples, mais pourraient se résumer au désir de s'affranchir de l'adolescence ou, à tout le moins, en atténuer les effets et à prendre sa place dans le monde pour faciliter le passage vers la vie adulte. 
Avoir 20 Ans en 2015 favorise "[...] le questionnement citoyen» (Bilodeau, 2012, p. 3) en amenant ces jeunes à prendre position, exprimer leurs opinions, confronter leurs idées, préciser leur pensée, réfléchir en s'ouvrant à l'altérité. Cet article témoigne du parcours réalisé au cours des quatre dernières années. Il marque un temps d'arrêt avant l'ultime étape du projet où ils franchiront alors le cap de leurs vingt ans.

\section{Méthodologie}

Dans le cadre d'une première analyse des retombées éducatives, nous tenterons de dégager quelques constats de cette expérience qui s'est poursuivie au cours des cinq dernières années. Cette analyse préliminaire repose sur des données recueillies lors de trois entretiens: deux collectifs, ont été réalisés avec le groupe des jeunes de Montréal et un troisième, individuel, a été fait avec la coordonnatrice du projet. D’autres données proviennent d'un questionnaire complété par les jeunes à la suite d'un entretien collectif. Nous avons également examiné et analysé différentes traces qui témoignent de cette expérience de médiation culturelle et théâtrale. Parmi ces traces, il y a l'enregistrement d'une conférence effectuée par des jeunes du groupe dans le cadre d'un forum sur la créativité, le dossier de présentation du projet, les bilans annuels des activités accomplies, le contenu d'un blog où les jeunes témoignent de leur parcours, la revue de presse et quelques entrevues individuelles (vidéos) qui avaient déjà été réalisées dans le cadre du projet. La diversité de ces sources reflète l'itinéraire et les aspirations des jeunes Montréalais, mais aussi la richesse et la complexité du dispositif qui a été conçu et mis en œuvre, afin de donner à ces jeunes un accès privilégié à l'art en leur faisant vivre une expérience remarquable. Ces données ont été traitées de manière globale au regard de catégories récurrentes qui ont émergé pendant les échanges, de la lecture et de l'analyse de ces traces issues de différentes sources. À la demande des jeunes, certaines réponses contenues dans les entretiens ou le questionnaire ne sont pas associées à leurs auteurs. Ces énoncés ont été retenus parce que la teneur et le sens véhiculés revenaient à plusieurs reprises et suscitaient souvent l'adhésion du groupe. Par contre, les extraits issus du blog sont clairement identifiés par leur auteur respectif puisque ces textes sont déjà publiés sur le Web. Ces premiers résultats soulignent l'importance de ce type d'expérience pour des jeunes en quête d'une identité et de son affirmation. 


\section{Sophocle}

Sophocle aura été un tremplin pour favoriser la discussion, le partage et «[...] le questionnement citoyen chez les jeunes» parce qu'il permet de s'interroger «[...] sur le monde d'aujourd'hui et de créer un espace [que les jeunes] pourront utiliser pour partager leurs réflexions et les créations inspirées par le contact avec ces œuvres» (Vigneault, 2001, p. 10).

Sophocle a toujours été en filigrane; il était présent dès les balbutiements du projet. «Par des voyages, des rencontres et des discussions philosophiques, le metteur en scène souhaite faire grandir ces jeunes jusqu'à l'âge adulte au contact de la pensée de Sophocle» (Beauregard, 2011, p. 53). La création du premier opus a été au centre du premier voyage. Rappelons que Wajdi Mouawad travaille tout au long du projet à la mise en scène des tragédies de Sophocle. L'expérience va se conclure en 2015 avec la présentation de l'intégrale à Mons (Belgique). À cette occasion, il y aura également une exposition qui fera une rétrospective du projet Avoir 20 Ans en 2015.

\section{Mise en Contexte d'un Dispositif de Médiation Théâtrale et Culturelle Complexe}

Dans les milieux institutionnels de l'art où elle est apparue, la médiation culturelle recouvre les dispositifs qui participent de la création des ouvres, de leur diffusion et de leur réception par les publics par le biais d'une éducation ou d'une animation. [...] Fondées sur des discours autorisés, leurs interventions se concentrent sur l'explicitation des conditions matérielles et techniques de production, le sens de la démarche et de l'intentionnalité des créateurs (Lafortune, 2012, p. 10).

Avoir 20 Ans en 2015 sollicite l'engagement des jeunes au regard de la création et de la réception des œuvres, mais aussi sur le plan de l'engagement citoyen. Il comprend quatre grands blocs d'activités: le parcours culturel, les questions soumises à la communauté, les rencontres avec Wajdi Mouawad et les voyages. Nous allons présenter sommairement chacune de ces grandes activités avant de passer à l'analyse en faisant ressortir les retombées du projet qui sont liées à l'expérience, à l'acte d'apprendre ainsi qu'à la fréquentation des œuvres. 


\section{Un Parcours Culturel}

Ce parcours comprend différentes sorties (théâtre, danse, musique, arts visuels ou évènements ${ }^{2}$ ), certaines sont obligatoires alors que d'autres sont suggérées et les jeunes sont libres de participer ou non. En plus de faire connaître l'offre montréalaise, ces sorties permettent de favoriser des rencontres avec des artistes d'ici ou d'ailleurs. La programmation peut également être influencée par les thèmes qui ont été associés aux voyages ${ }^{3}$. Parmi les activités du parcours, il y a aussi les Rendez-vous des Familles où les jeunes et leur famille sont invités aux générales du Théâtre du Nouveau Monde (TNM).

Si l'art est un moyen pour établir un dialogue entre les arts, le public, la communauté [...]. Dès lors, la stratégie de la médiation culturelle est à rechercher dans la configuration d'un itinéraire, dans une trajectoire de la personne qui ouvre sur une rencontre [...], stations par lesquelles passe le sujet pour gagner cette valeur spécifique qu'est sa culture. Il s'agit alors de ponts et de portes qui permettent de mettre en relation la personne avec l'autre, les autres et leurs cultures (Caune apud Lafortune, 2012, p. XIII).

Le parcours fait l'objet d'une préparation informelle où l'animatrice et coordonnatrice du projet, Claudia Bilodeau ${ }^{4}$, agit à titre de médiatrice culturelle. Sans fournir des clés de lecture explicites ou livrer des indications qui pourraient trop orienter le regard des jeunes spectateurs, elle communique certaines informations qui favorisent la compréhension et la réception de l'œuvre en la situant aussi parfois dans le contexte social, culturel et historique qui l'a vu naître.

Après un spectacle, les jeunes font un bilan sommaire, mais la discussion se fera lors d'une prochaine rencontre au cours de laquelle ils pourront se le remémorer, exprimer ce qu'ils ont ressenti et construire ensemble le sens de l'œuvre, etc. Entre les deux, les jeunes peuvent également témoigner de la réception qu'ils ont faite de l'œuvre dans le blog ${ }^{5}$ du projet.

Depuis le début de cette entreprise, les adultes accompagnants observent qu'ils se sont ouverts à diverses propositions, que leur regard de spectateur s'est affiné, leur discours s'est enrichi de tous les spectacles qu' ils ont vus ${ }^{6}$. Ils sont devenus des spectateurs aguerris en développant leur jugement esthétique et critique. Ils ont développé leur habileté à apprécier une œuvre, ils sont capables d'en discuter avec d'autres, de confronter leur vision à celles des autres, d'appuyer leur propos en utilisant un vocabulaire approprié. 


\section{Aller à la Rencontre de l'Autre}

Cette activité a été la plus difficile à réaliser: certaines villes qui participent aux projets l'ont abandonnée en cours de route. Cependant, même si les jeunes de Montréal s'accrochent puisqu'ils croient à la pertinence de l'exercice, ils peinent à le faire. Initialement, ils devaient interroger une personne inconnue. Au terme d'une année, ils devaient avoir posé sept questions différentes à des personnes de leur communauté, puis recommencer la séquence de questions d'une année à l'autre. Ces questions ont trait à la capacité d'étonnement, au sens de la vie, à ce qui nous fait peur, à l'héritage transmis par les parents, à ce que veut dire réussir sa vie etc.

À la défense des jeunes, il faut dire que les questions ne sont pas nécessairement faciles à traiter avec des étrangers. La difficulté aurait probablement été aplanie s'ils avaient pu poser ces questions à des membres de leur entourage, ou à d'autres jeunes, ou s'ils avaient participé à la formulation des questions. Ce qu'ils ont fait, avec vraisemblablement beaucoup de candeur à quinze ans, s'est avéré avec le temps de plus en plus difficile à faire. Plusieurs jeunes expriment leur malaise à interroger un adulte sur le sens de sa vie, même si les questions étaient formulées de manière indirecte.

\section{Rencontre Individuelle avec un Créateur}

En plus d'être présent et de les accompagner lors de leurs séjours à l'étranger, Wajdi Mouawad rencontre individuellement tous les jeunes chaque année. Au cours de ces entretiens, ils parlent de leurs aspirations et de leurs rêves, de la création théâtrale, des apprentissages qu'ils font à travers ce projet. Au début, plusieurs se disaient intimidés par ces échanges avec un créateur de la stature de Mouawad, mais la gêne a rapidement cédé le pas au plaisir de discuter avec l'homme et l'artiste.

Dans une perspective de médiation culturelle, d'accessibilité à l'art et à la culture, ce type de relations illustre la responsabilité et le rôle que l'artiste peut jouer dans la valorisation et la transmission de pratiques artistiques et culturelles. Pour ces jeunes, c'est un interlocuteur crédible. 


\section{Les Voyages}

Une fois par an, les cinquante jeunes se retrouvent pour un séjour d'une semaine à l'étranger. Chaque voyage s'organise autour d'une thématique qui guide le choix des activités au programme, le choix des accompagnateurs et les personnes rencontrées. Ces thématiques sont inspirées par une réplique d'un personnage de Mouawad où une grand-mère dit à sa petite fille que pour survivre et réussir sa vie, elle doit apprendre à lire, à écrire, à compter, à parler et à penser.

Les voyages comptent parmi les moments mémorables du projet. Ils constituent un puissant révélateur qui amène les jeunes à apprécier et à considérer autrement leur culture en étant confronté à celles des autres: celle du pays hôte, mais aussi celle des autres participants originaires de pays différents. Le premier séjour a été particulièrement déstabilisant, il a donné le ton de cette grande aventure où ils devaient à quinze ans apprivoiser une liberté nouvelle. Pour la plupart, il s'agissait d'un premier voyage avec d'autres personnes que les membres de leur famille.

\section{Apprendre à Lire à Athènes (Grèce, 2011)}

Au cours de ce premier voyage, les jeunes assistent à la représentation Des femmes, le premier opus du Projet Sophocle, au théâtre Hérode Atticus. Ils gardent un souvenir impérissable de cette trilogie, de la discussion qui a suivi la représentation avec l'équipe de production, des chiens errants qui hurlent, de la crise économique, des manifestations, des moments partagés avec les autres jeunes du projet qu'ils rencontrent pour la première fois. Ils étaient accompagnés par Constantin Bobas, helléniste, Robert Davreu, le traducteur de la Trilogie des femmes, François Ismert, le dramaturge et Wajdi Mouawad, le metteur en scène. Ils ont visité le quartier de l'Acropole, le port du Pirée, Marathon, le temple d'Apollon et l'Épidaure.

\section{Apprendre à Écrire à Lyon (France, 2012)}

Consacré à l'écriture, ce deuxième voyage invite à "[...] la découverte de l'histoire de l'imprimerie lyonnaise et des métiers qui se rapportent aux livres» (Bilodeau, 2013b, p. 4). Les matinées sont consacrées à des ateliers d'écriture avec Mouawad aux Subsistances ${ }^{7}$, ils sont suivis de conférences, de visites et de rencontres. 
Wajdi a ouvert un chemin qui nous amène à la fois à comprendre un parcours personnel menant à l'écriture, le sien, mais également à une invitation à découvrir une route à part, la nôtre. Ainsi, les récits divers s'entrecoupaient d'exercices concrets [...] (Théâtre de Nouveau Monde, 2014, n.p.).

Pour les jeunes, ce voyage est celui qui les a le plus sollicités sur le plan cognitif, mais ils précisent que c'est à ce moment qu'ils sont devenus de véritables amis.

Accompagnés par Nathalie Zémon-Davis, historienne et spécialiste de l'histoire culturelle et sociale de France, les activités avaient été choisies au regard de l'importance de l'imprimerie et du rôle de l'écrit dans la résistance. Au cours de ce voyage, les jeunes ont aussi visité «[...] différents lieux de culte et se sont interrogés sur les fondements de grandes religions monothéistes» (Bilodeau, 2013b, p. 4). Les lieux visités sont le Vieux Lyon, le Musée de l'imprimerie, le Grand Temple, la synagogue du quai Tilsitt, la bibliothèque de la Part-Dieu.

\section{Apprendre à Compter à Auschwitz (Pologne, 2013)}

Ce troisième séjour s'inscrit dans un devoir de mémoire: «À travers ce récit de la Shoah où les nombres se déploient dans des dimensions toutes particulières, il nous est possible de comprendre que derrière les chiffres, il y a des gens» (Théâtre de Nouveau Monde, 2014 , n.p.). Ce voyage se caractérise par son intensité: une préparation fébrile de la visite du camp, puis l'impression de se retrouver face à un décor appartenant à une autre époque, à une réalité loin de la leur. Ils sont stupéfaits de voir que tout a été reconstruit, nettoyé. Ils ne s'attendaient pas à cela. La visite aurait été incomplète s'ils n'avaient pas rencontré des survivants pour témoigner de ce qui s'était passé. Ils ont été habités par un maelström d'émotions face à ce devoir de mémoire auquel ils arrivent difficilement à s'identifier. Face à l'indicible, ils expriment leur difficulté à jouir du voyage, à s'autoriser à vivre.

Ils sont accompagnés par Arthur Nauzyciel, metteur en scène et petit-fils de déporté, Sophie Ernst, professeur de philosophie et spécialiste de la pédagogie de la Shoah, et Éric Ghozan, psychologue-psychanalyste. Les lieux visités sont le Musée de l'histoire du judaïsme, le mémorial de la Shoah, le ghetto de Varsovie, Auschwitz. 


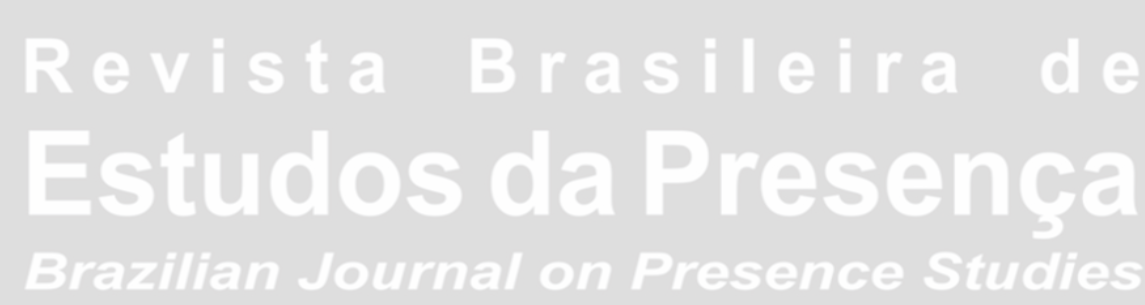

Apprendre à Parler à Toubab Dialaw (Sénégal, 2014)

Riche d'une tradition orale de transmission des valeurs et de la culture, le Sénégal est tout indiqué pour ce quatrième voyage. Ils sont accompagnés par Lilian Thuram, ex-footballeur international. Ils logent dans un centre d'artistes au bord de l'océan. Au programme des ateliers d'écriture et de jeu avec Wajdi Mouawad, du foot, des échanges avec des artistes sénégalais, un débat sur l'Afrique contemporaine. Ils visitent Dakar, l'Institut de Gorée, la Maison des esclaves et l'île de Gorée.

\section{Apprendre à Penser (2015)}

L'itinéraire du cinquième voyage n'est pas encore arrêté, mais comme il marquera la fin du projet, ils seront appelés à vivre une expérience qui valorisera la responsabilisation et l'autonomie des participants de manière à donner à chacun le lest nécessaire pour poursuivre sa vie avec un petit coquillage au fond de sa poche ${ }^{8}$.

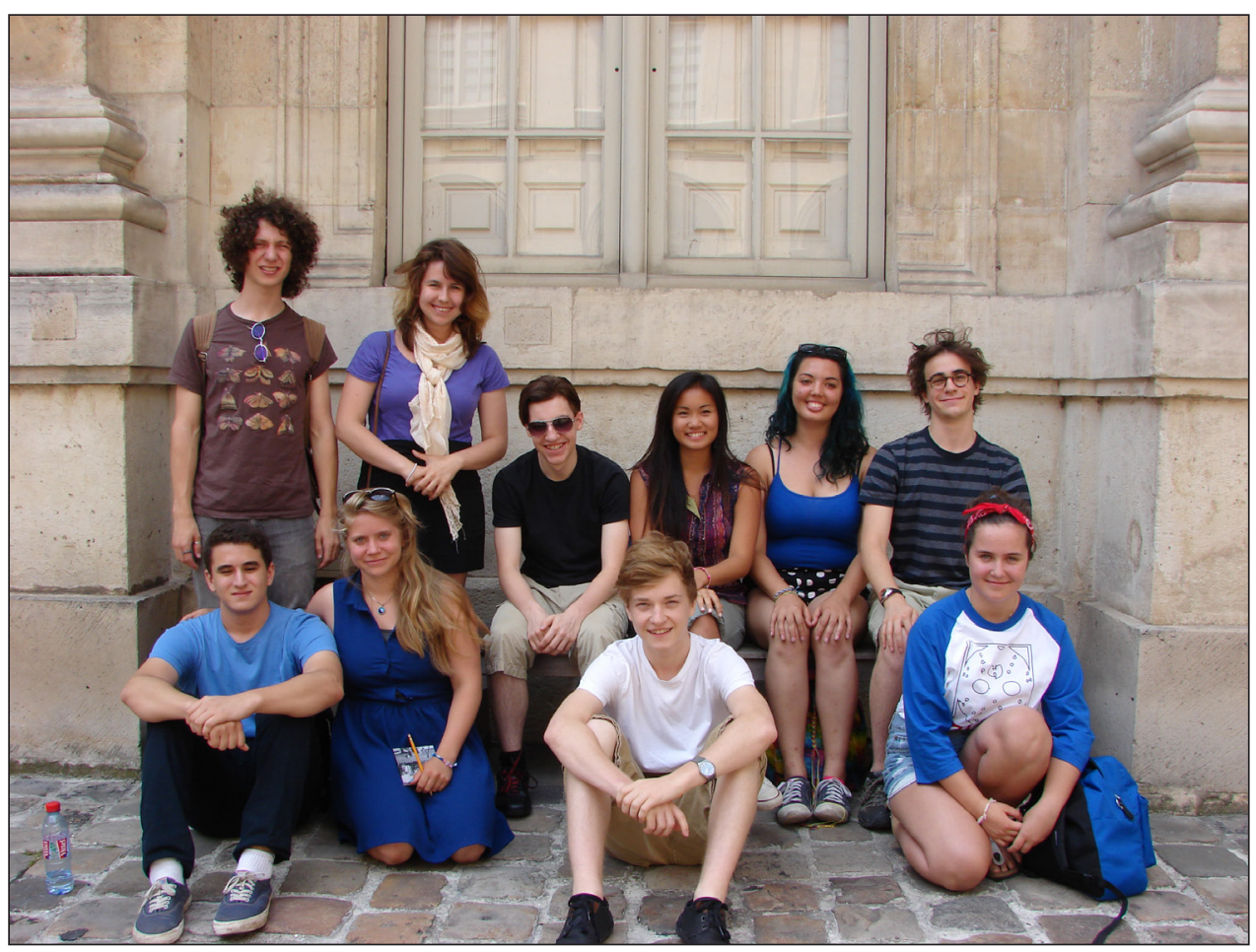

Image 1 - Les dix jeunes montréalais qui participent au projet Avoir 20 Ans en 2015, photographiés à Montréal. De gauche à droite, de bas en haut: Nadjib Bouazouni, Anne-Marie Saint-Louis, Vladimir Belova, Natasha Beaudin-Pearson, Benjamin Charette, Juliane Choquette Lelarge, Quentin Gagnon, Justine Le Minh Hien, Éléonore Brieuc, Alexis Curodeau-Codere. Photo: Claudia Bilodeau. 


\section{Retombées de cette Expérience de Médiation Théâtrale et Culturelle}

Ce projet est une occasion de s'ouvrir à soi, de partir à la rencontre de l'autre, d'apprivoiser différentes réalités, de respecter la diversité, de s'ouvrir à d'autres valeurs que les siennes et de développer un sentiment d'appartenance à une communauté. Le choix des activités et la composition du groupe confrontent et préparent les jeunes aux exigences du vivre ensemble et à l'exercice de la citoyenneté.

Le débat sur la diversité qui s'en suit situe la culture, en tant que moyen de communication entre les membres des groupes socioéconomiques et des communautés ethnoculturelles, au cœur des stratégies visant à renforcer le sentiment d'appartenance et à stimuler l'adhésion à une identité commune (Lafortune, 2012, p. 11).

Même s'ils étaient très motivés pour participer, une fois engagés, ils réalisent rapidement l'impact qu'aura ce projet. Ils ne savent pas encore comment cela va se traduire d'ici cinq ou dix ans, mais ils s'accordent pour dire que cette entreprise a grandement contribué au développement de leur personnalité. Ils peuvent déjà identifier certains changements dans leur manière d'être, de réfléchir, de se poser des questions, etc. Ils constatent avoir une meilleure compréhension d'eux-mêmes, de ce qu'ils sont et de ce qu'ils souhaitent devenir.

Pour certains, la perspective de participer à ce projet permet d'échapper au cynisme ambiant, de cultiver l'espoir. Il a aussi changé leurs perceptions. Ils ont le sentiment de pouvoir faire une différence dans la vie des autres, d'avoir une prise sur le cours des évènements. Les jeunes ont fait l'expérience de la solidarité humaine et sont rassurés de savoir qu'ils ne seront jamais seuls face à l'adversité.

Cette aventure les incite grandement à réfléchir sur le sens du don et sur la capacité à recevoir des autres. Le projet les amène à relativiser leurs valeurs, à savoir que le don peut être un acte gratuit. Aujourd'hui, ils sont moins méfiants face à la générosité, ils ont appris à ne pas la considérer comme étant suspecte ou intéressée.

Lorsqu'on leur demande d'envisager un futur proche, les jeunes font preuve d'une solide assurance. De leur adolescence, ils souhaitent conserver certaines attitudes comme l'ouverture, l'authenticité, la créativité, la passion, l'indépendance, la capacité de s'émerveiller, mais aussi le pouvoir de s'indigner par rapport à certaines situations. Pour Claudia, la coordonnatrice du projet, cette expérience aura 
permis à ces jeunes de se révéler et de confirmer ce qu'ils étaient déjà comme individu.

\section{Sur l'Apprentissage, l'Éducation}

Au cours des échanges, les jeunes évoquent souvent l'idée d'apprendre autrement ou d'apprendre par soi-même, à travers l'expérience: «On peut tenter d'expliquer, de faire comprendre, partager des bribes de nos connaissances et de nos expériences, mais il reste que certaines choses doivent être vécues». Le projet, surtout les voyages, place ces jeunes en situation de vivre des expériences concrètes, ils sont confrontés à des situations réelles où ils doivent faire preuve d'une autonomie grandissante.

À propos d'apprendre autrement, Lorraine Pintal, directrice artistique du Théâtre du Nouveau Monde, un des partenaires du projet, préfère parler d'apprentissage plutôt que de pédagogie:

[...] apprentissage qui me semble inclure plus de ludisme, de liberté et de plaisir. On n'est pas dans la hiérarchie du savoir. Pour nous c'est une façon de rejoindre les jeunes, de montrer que l'art n'est pas inaccessible. L'école ne remplit pas toujours son rôle en ce sens (Lalonde, 2011, p. B7).

Pour certains jeunes, l'école est un lieu qu'ils traversent comme un désert sans fin. Un lieu de transmission où l'on accumule passivement des connaissances. Ils déplorent que le curriculum scolaire mise trop sur l'accumulation de savoirs et de savoir-faire, mais qu'elle ne favorise pas assez la construction de savoir-être. À la manière de Montaigne, ils préféreraient avoir une tête bien faite plutôt que d'avoir une tête bien pleine. Ils s'interrogent: l'art et la culture peuvent-ils enseigner quelque chose que l'école ne peut pas leur offrir? L'école ne devrait-elle pas être le meilleur foyer d'intégration des valeurs culturelles d'une société?

Ils ajoutent que l'école ne les prépare pas à la résistance, elle ne valorise pas ceux qui vont à contre-courant. Ils revendiquent le droit à la différence et désirent que leurs voix soient entendues, même lorsqu'ils s'écartent des sentiers battus. Ils déplorent que l'art soit souvent perçu comme étant subversif par l'école qui est bien souvent un lieu de conformité sociale où l'on apprend à entrer dans les rangs.

En s'ouvrant aux expériences des autres, ils apprennent à se faire confiance, à faire confiance aux autres, à développer une meilleure 
écoute, ils sont plus accueillants, plus tolérants, plus conscients des autres et de leur environnement. Ce projet «[...] m’a appris à m’ouvrir à des opinions contraires à la mienne et à les accepter, il m’amène à mieux comprendre des personnes que je n'aurais jamais croisées autrement».

En quelques jours, le projet a créé un «nous` avec des individualités qui ne se seraient jamais rencontrées, explorées sinon, et qui soudainement forment l'unité la plus forte. En fait, je crois que si ce qu'on vit est aussi puissant, c'est parce qu'en plus d'apprendre à lire, à écrire, à compter, à parler et à penser, nous apprenons à être ensemble. À rejoindre l'Autre. Et il n'y a rien de plus beau que de se dire que malgré tout, malgré les kilomètres, les mois, les obligations, nous serons toujours «nous». Sur une plage à Athènes comme dans une cuisine à Lyon comme dans un camp de concentration [...]. Trois ans plus tard [...] je me rends compte que vous n'êtes pas une expérience de jeunesse ou un passage rituel. Vous n'êtes pas mes 15-20 ans. Vous n'êtes pas le mot (projet). Vous êtes une promesse qui se fait sur toute une vie et qui ne se brisera pas simplement en 2015 (Théâtre de Nouveau Monde, 2014, n.p.).

\section{Retombées liées à la Fréquentation des Euvres}

Pour la majorité de ces jeunes, l'art et la culture sont indissociables et interdépendants, ils s'enrichissent mutuellement. Pour l'un des jeunes, la «[...] culture définit la société et l'art en est l'expression la plus directe [...] connaître l'art, c'est connaître la société».

Les œuvres théâtrales ne sont jamais anodines ou gratuites, elles parlent toutes de l'énigme de la vie, de la recherche de la vérité, de notre impossible travail d'humanité. Elles parlent la langue de ce ssavoir sans connaissance〉 que nous portons tous sur notre dos: elles nous aident parfois à le porter (Soussan apud Planson, 2008, p. 16).

Ils réalisent que l'art n'est pas uniquement un produit de consommation ni un simple divertissement, mais qu'il peut aussi être nécessaire à l'épanouissement des personnes qui le fréquentent.

À propos d'un spectacle de Fred Pellerin", Éléonore dit: «Je me surprends à adorer l'idée que les gens aiment se faire raconter des histoires. On oublie trop souvent la nécessité de la poésie, les bienfaits qu'elle nous apporte» (Théâtre de Nouveau Monde, 2014, n.p.).

Les jeunes soulignent l'importance de faire la promotion de l'art, de le rendre accessible. Pour eux, l'art ne devrait pas être ré- 
servé à une élite. Ils constatent qu'on peut apprendre de l'art, car il tend «[...] un miroir au monde, calque ou déforme son image. L'art renvoie dans la gueule de la société, tout ce qui ne va pas, masque, décore et esthétise la réalité afin qu'elle retentisse avec plus de clarté». L'art peut être irrévérencieux, il nous fait voir nos travers, nous aide à vivre avec nos paradoxes. Il parle de ce que nous sommes, de ce qui nous anime, nous aide à évoluer. En parlant d'une pièce de Dennis Kelly ${ }^{10}$, Juliane exprime bien la force de frappe que certaines pièces peuvent exercer sur les spectateurs:

Coup de poing. Coup dans les flans, souffle coupé, mal à respirer, bulle d'anxiété, identification douloureuse, vertige du non-retour, catharsis, fiction-qui-frappe-trop-près, fiction-qui-gueule-trop-vrai, boum, en plein dans le plexus. Première fois que je sors d'un spectacle avec une cage thoracique explosée (Théâtre de Nouveau Monde, 2014, n.p.).

L'art est nécessaire à l'expression des individus, d'une culture, mais c'est aussi un patrimoine qu'il faut protéger. Face à la qualité des productions auxquelles ils ont assisté, un des jeunes déplore le peu d'importance que l'on accorde aux manifestations de l'art dans nos sociétés. Les autres renchérissent en ajoutant qu'ils sont rarement encouragés à faire un métier artistique.

Ils soulignent également l'importance de s'ouvrir à d'autres cultures, à d'autres visions du monde. Si l'art et la culture sont le reflet d'une société, il importe de s'ouvrir à l'expérience des autres pour mieux vivre ensemble. La composition du groupe est également un modèle d'intégration, puisque plusieurs jeunes du projet de Montréal sont issus de communautés culturelles diverses. L'effet d'ouverture en est multiplié, comme lorsqu'ils rencontrent des cultures différentes lors des séjours à l'étranger.

Si l'heure n'est pas encore au bilan, les jeunes réalisent la valeur du trésor qui leur a été confié à travers ce projet. Ils sont reconnaissants et mesurent tout le chemin qui a été parcouru au cours des dernières années. Ils ont été surpris de n’avoir rien eu à payer en retour et cela les a amenés à s'interroger sur la capacité à recevoir. Ils souhaitent pouvoir transmettre à d'autres une petite part de ce qu'ils ont reçu à travers l'expérience.

Avoir 20 Ans en 2015 est un projet exemplaire par la complexité du dispositif qui a été mis en place, afin de faire vivre à des jeunes des expériences liées à la fréquentation des œuvres sur un continuum 
de plusieurs années. Outre son étendue dans le temps, ce projet se distingue par la diversité et la richesse des activités qui ont été proposées aux jeunes, mais aussi par le choix des personnes qui les ont accompagnés tout au long de cette aventure. Pendant cinq ans, dix jeunes ont été invités à fréquenter des œuvres à une époque de leur vie où ils sont confrontés à plusieurs changements. «Fondée sur des processus de transmission et d'appropriation de la culture [...]» (Lafortune, 2012, p. 211), cette expérience a réuni des jeunes issus de différents milieux.

Au départ, ces jeunes ne se connaissaient pas, comme nous l'avons écrit plus tôt, ils ont été choisis parmi une centaine de candidats à partir d'une ouvre et d'une entrevue, mais ce qu'on retient du groupe, même si les individus sont très différents les uns des autres, c'est la cohésion, la solidarité et le sentiment d'appartenance qu'ils ont développés tout au long de ce parcours. Il s'agit de personnes curieuses, très ouvertes aux autres et aux expériences qu'on leur propose. Au début du projet, ils étudiaient en deuxième cycle du secondaire, depuis ils se sont engagés dans des études supérieures (Diplôme d'études collégiales). Sur les 10 jeunes, plus de la moitié sont actuellement inscrits dans un parcours de formation qui est en lien avec une discipline artistique: création littéraire, théâtre, musique, ou font un double cheminement en arts et sciences. Est-ce le projet qui a influencé ce choix ou avaient-t-ils déjà un intérêt manifeste pour l'art et la culture? On ne saura jamais ce qu'il serait advenu sans ce projet, mais on peut supposer qu'ils ont été sélectionnés à cause de leur sensibilité pour l'art. Cette expérience a certainement été un révélateur permettant à ces jeunes d'être en contact avec des modèles forts, un merveilleux prétexte pour faire rêver de jeunes citoyens. 


\section{Notes}

${ }^{1}$ Parallèlement à Avoir 20 Ans en 2015, Wajdi Mouawad travaille sur le Projet Sophocle, une création franco-québécoise qui sera créée en trois temps: le premier opus, Des Femmes (Les Trachiniennes, Antigone et Électre) a été présenté en 2011, il sera suivi Des Héros (Ajax et Edipe Roi) et Des mourants (Edipe à Colone et Philoctète).

${ }^{2}$ Parmi ces évènements, ils ont entre autres travaillé à une activité de médiation culturelle avec une artiste et des sans-abri qui réalisait une ouvre collective dans le cadre de Fin Novembre, Dormir Dehors organisé par l'Association Terroriste Socialement Acceptable (ATSA).

${ }^{3}$ Ces thèmes sont présentés plus loin dans le texte.

${ }^{4}$ En plus d'animer les activités du groupe et de les accompagner lors des voyages, Claudia Bilodeau fait actuellement une maîtrise sur l'accompagnement du processus de création chez les non-acteurs.

${ }^{5}$ On peut consulter ce blog en se rendant à l'adresse suivante: <http://www.tnm.qc.ca/ tout-sur-le-tnm/avoir_20ans.html>.

${ }^{6}$ Annuellement, ils assistent à une dizaine de spectacles obligatoires.

${ }^{7}$ Il s'agit d'un laboratoire international de création artistique.

${ }^{8}$ Face à l'ennui, aux passages à vide ou à la difficulté à réintégrer ses activités quotidiennes au retour d'un voyage, Wajdi Mouawad a dit aux jeunes qu'il mettait sa main au fond de sa poche et qu'il se disait à lui-même cette petite phrase pour garder intact le souvenir de l'expérience vécue et faire face à l'adversité.

${ }^{9}$ Fred Pellerin, conteur québécois, a présenté son spectacle Depeigne et de misère, au Théâtre Maisonneuve, le 11 avril 2014.

${ }^{10}$ La pièce Comment s'Occuper de Bébé a été présentée au Théâtre La Licorne, le 21 mars 2014.

\section{Références}

BEAUREGARD, Martin. La Chance de Grandir avec l'Euvre de Sophocle. Journal de Montréal, Montréal, p. 53, juin 2011.

BÉLAIR, Michel. Côté Jardin puis Côté Cour (d'Honneur). Le Devoir, Québec, juin 2011. Culture, p. B7.

BILODEAU, Claudia. Avoir 20 Ans en 2015: le bilan annuel des activités du projet, 2011-2012. Montréal: [s.n.], 2012.

BILODEAU, Claudia. Avoir 20 Ans en 2015: le bilan annuel des activités du projet, 2012-2013. Montréal: [s.n.], 2013d.

BILODEAU, Claudia. Avoir 20 Ans en 2015: le projet - les détails. Montréal: [s.n.], 2013b. 
BILODEAU, Claudia. Avoir 20 Ans en 2015: le projet en bref. Montréal: [s.n.], 2013a. BILODEAU, Claudia. Avoir 20 Ans en 2015: la revue de presse. Montréal: [s.n.], 2013c. COLPÉ, Chloé. Entrevues Individuelles Réalisées avec les Jeunes Montréalais. Belgique: Université de Mons, 2014.

FORUM CRÉATIVITÉ QUÉBEC. Home Page. Québec, 2014. Conférence donnée par des jeunes du groupe de Montréal. Disponible sur: <http://forumcreativite.com/>. Consulté le: 25 mai 2014.

LAFORTUNE, Jean-Marie (Dir.). La Médiation Culturelle: le sens des mots et l'essence des pratiques. Préface: Jean Caune. Montréal: Presses de l'Université du Québec, 2012.

LALONDE, Catherine. Avoir 20 Ans en 2015: l'apprentissage. Le Devoir, Montréal, juin 2011. Culture, p. B7. Disponible sur: <http://www.ledevoir.com/culture/theatre/326501/ avoir-20-ans-en-2015-l-apprentissage>. Consulté le: 24 mai 2014.

PETROWSKI, Nathalie. Vladimir qui Aura 20 Ans en 2015. La Presse, Montréal, 04 juin 2011. Arts et Spectacles, p. 20.

PLANSON, Cyrille, Accompagner l'Enfant dans sa Découverte du Spectacle. Condésur-Noireau: La Scène, 2008.

THÉÂTRE DE NOUVEAU MONDE. Le Blogue d'Avoir 20 Ans en 2015. Québec, 2014. Disponible sur: <http://www.tnm.qc.ca/a-surveiller/Le-blogue-Avoir-20-ansen-2015/>. Consulté le: 05 juil. 2014.

VIGNEAULT, Alexandre. Avoir 20 Ans en 2015, Mouawad comme Guide. La Presse, Québec, juin 2011. Arts et Spectacles, p. 10.

Chantale Lepage est professeur à l'École supérieure de théâtre de l'Université du Québec à Montréal. Elle contribue à la formation des enseignants en art dramatique. Elle a réalisé plusieurs mandats pour le ministère de l'Éducation. Sur le plan de la recherche, elle s'intéresse à l'accompagnement des pratiques de formation en arts, à l'intégration de la dimension culturelle à l'école et à la dramaturgie scolaire.

E-mail: lepage.chantale@uqam.ca

Ce texte inédit, révisé par Annelyse Gayraud, est également publié en portugais dans ce numéro. 